\title{
Small-cell lung cancer patients are just 'a little bit' tired: response shift and self-presentation in the measurement of fatigue
}

\author{
Marjan J. Westerman · Anne-Mei The • \\ Mirjam A. G. Sprangers · Harry J. M. Groen • \\ Gerrit van der Wal · Tony Hak
}

Received: 2 November 2006/ Accepted: 16 January 2007/Published online: 15 February 2007

(C) Springer Science+Business Media B.V. 2007

\begin{abstract}
Background Response shift has gained increasing attention in the measurement of health-related quality of life (QoL) as it may explain counter-intuitive findings as a result of adaptation to deteriorating health.

Objective To search for response shift type explanations to account for counter-intuitive findings in QoL measurement.

Methods Qualitative investigation of the response behaviour of small-cell lung cancer (SCLC) patients $(n=23)$ in the measurement of fatigue with The European Organization for Research and Treatment of Cancer Core Quality of Life Questionnaire (EORTC QLQ-C30) question 'were you tired'. Interviews were conducted at four points during 1st line chemotherapy: at the start of chemotherapy, 4 weeks later, at the end of chemotherapy, and 6 weeks later. Patients were asked to 'think aloud' when filling in the questionnaire. Results Fifteen patients showed discrepancies between their answer to the EORTC question 'were you tired' and their level of fatigue spontaneously reported
\end{abstract}

M. J. Westerman $(\bowtie) \cdot$ A.-M. The · G. van der Wal Department of Public and Occupational Health, EMGO Institute, VU University Medical Centre, Van der

Boechorststraat 7, 1081 BT Amsterdam, The Netherlands e-mail: MJ.Westerman@vumc.nl

\section{A. G. Sprangers}

Department of Medical Psychology, Academic Medical

Centre, Amsterdam, The Netherlands

\section{H. J. M. Groen}

Department of Pulmonary Diseases, University Hospital, Groningen, The Netherlands

T. Hak

RSM, Erasmus University, Rotterdam, The Netherlands during the interview. These patients chose the response options 'not at all' or 'a little' and explained their answers in various ways. In patients with and without discrepancies, we found indications of recalibration response shift (e.g. using a different comparison standard over time) and of change in perspective (e.g. change towards a more optimistic perspective). Patients in the discrepancy group reported spontaneously how they dealt with diagnosis and treatment, i.e. by adopting protective and assertive behaviour and by fighting the stigma. They distanced themselves from the image of the stereotypical cancer patient and presented themselves as not suffering and accepting fatigue as consequence of treatment.

Conclusion In addition to response shift, this study suggests that 'self-presentation' might be an important mechanism affecting QoL measurement, particularly during phases when a new equilibrium needs to be found.

Keywords Small-cell lung cancer - Quality of life . Response shift · Self-presentation · Fatigue

\section{Introduction}

Quality of life (QoL) is considered an important treatment outcome when the treatment intent is not curative but palliative. However, the expected deterioration in QoL often does not occur, even in cases of serious illness. For example, Groen et al. studied patients with inoperable non-small cell lung cancer treated by radiation with and without chemotherapy [7]. QoL was measured with The European Organization for Research and Treatment of Cancer Core Quality of Life Questionnaire (EORTC QLQ-C30) [1], which has 
been designed specifically for use in clinical trials focusing on cancer patients. However, contrary to expectation, they did not find significant deterioration in the scale scores over the treatment period of 6 weeks. Although cancer patients are willing to undergo risky and toxic treatments [11], it seemed implausible that the side-effects of treatment had not affected their QoL. Other studies also reported counter-intuitive results. For example, patients with a lifethreatening disease or disability were found to report stable QoL, and patients with a severe chronic illness reported QoL levels that were not inferior to that of patients with a less severe illness or to healthy patients $[2,3,5]$. Such counter-intuitive findings, labelled by Breetvelt and Van Dam as 'underreporting of problems' suggest that patients report less distress and dissatisfaction than they actually feel [5].

In recent years, response shift theory has gained increasing attention in explaining paradoxical and counter-intuitive findings. Response shift refers to a change in internal standards, values and conceptualization of QoL and is recognized as an important mediator in adaptation to changing health [17]. However, despite the explanatory power of response shift theory, our current understanding of phenomena that can complicate the interpretation of QoL scores is still limited. Therefore, we investigated QoL measurement in small-cell lung cancer (SCLC) patients during 1st line chemotherapy. We were quite surprised when we noticed discrepancies between levels of fatigue measured with the questionnaire and answers spontaneously reported during the interview. We therefore investigated these 'conflicting' findings in the measurement of QoL in more depth.

This paper reports the results of an exploratory longitudinal multiple-case study, in which we focused on how patients responded to the EORTC QLQ-C30 question 'were you tired' at different points in their treatment trajectory. We aimed to describe the patients' explanations when answering the question, and to search for explanations of counter-intuitive findings.

\section{Methods}

Procedures and study sample

Between March 2001 and September 2003, we recruited newly-diagnosed patients with SCLC who were evaluated for 1 st line chemotherapy. The patients were attending one of five outpatient clinics for chest diseases in the Netherlands. To maximize the likelihood that we would interview patients from the beginning of their treatment, we were informed about new patients immediately after diagnosis. No restrictions were made with regard to age or treatment (chemotherapy or a combination of chemotherapy and radiotherapy). Participating patients gave written consent and were interviewed at equivalent points in the treatment trajectory. The first interview (T1) was carried out at the start of chemotherapy. In the original plan the second interview was planned after completion of the course of chemotherapy. However, after inclusion and first interviews of 3 patients we made a decision to interview the patients during treatment as well. Therefore the second interview (T2) was conducted 4 weeks after $\mathrm{T} 1$ and the third (T3) 7-10 days after completion of the treatment with chemotherapy and the fourth (T4) 6 weeks later. Approval for this study was obtained from the Medical Ethics Committees of the research site and the participating hospitals.

During the course of the study, 41 eligible patients were invited to the study. Four patients were unwilling to participate, 3 died before informed consent could be obtained, and 3 were not interviewed because of imminent death. Of the 31 respondents who were interviewed, 8 were excluded from further analysis because their data were incomplete, i.e. they were only interviewed once (six died within a month after T1 and two were too sick at $\mathrm{T} 2$ and died before the end of the planned chemotherapy). Consequently, the final study sample consisted of 23 SCLC patients, of whom 12 had limited ( 3 male and 9 female, mean age 55, range 42 $69)$ and 11 had extended disease ( 8 male and 3 female, mean age 64, range 39-72). All patients received standard chemotherapy, except for 7 patients whose chemotherapy was combined with local radiation of the tumour. The majority of the patients were married $(19,83 \%)$, and had children $(17,74 \%)$.

Of the 23 patients in our study sample, 15 were interviewed four times, 7 were interviewed three times, and one patient was only interviewed twice resulting in a total of 83 interviews. The interviews were conducted by MW in the homes of the patients. In three cases the 1st interview was held in the hospital. Interviews averaged $80-110 \mathrm{~min}$.

\section{Materials and qualitative method}

In this exploratory, longitudinal multiple-case study, QoL was assessed with EORTC QLQ-C30 (version 3.0) [1] and the lung cancer module QLQ-CL13 [4]. The EORTC QLQ-C30 is the most widely used cancerspecific QoL instrument in European clinical trials. The questionnaire is composed of several scales, which measure among others physical function, mental 
health, general health and global QoL. Furthermore, it measures different symptoms such as pain, dyspnoea, nausea and fatigue. The Fatigue Scale consists of 3 items: 'did you need to rest', 'have you felt weak' and 'were you tired' (respectively questions 10, 12 and 18), for which there are 4 response categories: 'not at all', 'a little', 'quite a bit' and 'very much' (respectively scores $1,2,3$ and 4).

The EORTC QLQ-C30 and CL13 were completed in combination with the Three-Step Test-Interview (TSTI) to investigate how respondents interpreted the items and how they responded to them. The TSTI consists of the following steps [8]: (1) concurrent think aloud, aimed at collecting observational data on how a respondent completes the questionnaire, expressing his thoughts aloud; (2) focused interview, aimed at clarifying respondents' previous expressions while completing the questionnaire; (3) semi-structured interview, aimed at eliciting respondents' experiences and opinions with regard to the questionnaire.

\section{Interview protocol}

Each interview was conducted in an identical format. At T1, QoL was measured with the EORTC QLQC30, followed by the lung cancer module QLQ-CL13. The questionnaire was conducted in a concurrent think aloud manner and after completion, respondents were asked to clarify previous hesitations, expressions when rating certain items and experiences (i.e. second and third step of the TSTI). Individual QoL was then measured with the Schedule for the Evaluation of Individual Quality of Life-Direct Weighting (SEIQoL-DW) [13, 14]. Finally, we encouraged patients to talk freely about the impact of diagnosis and treatment. At follow-up (i.e. T2, T3 and T4), after the SEIQoLDW assessment, the EORTC questionnaires were administered a second time as a so-called 'then-test' $[15,16,18]$ (i.e. the patients filled out the questionnaire in reference to how they perceived themselves as they were in the previous interview). In these 2nd and following interviews, EORTC assessments were conducted in a concurrent think aloud manner and with the second step of TSTI integrated in the assessment. In fact, we encouraged patients to think aloud and we probed for clarification after each item in the case of extra information was considered useful to understand patients' answer. We used a flexible approach in order not to interrupt the natural flow of both the assessment as well as the patient-interviewer communication.

The interviews were audio-taped and transcribed verbatim. In this article we focus on the EORTC QLQC30 question 'were you tired'.
Analysis

Three types of data were collected and used for analysis [12]: (1) completed EORTC questionnaires (T1-T4), (2) observed respondent behaviour recorded in field notes, and (3) transcriptions of the interviews, including 'think aloud'. The analysis was aimed at identifying discrepancies, response strategies and explanations of response behaviour. We used the qualitative computer package Kwalitan 5.0 (http://www.kwalitan.net) to extract relevant parts of the transcriptions (1) 'think aloud' of the question 'were you tired' and of other items that were useful in understanding the response behaviour to the question on fatigue (i.e. two other items of the fatigue scale, the general health and the global QoL question of the QLQ-C30), and (2) 'comments' related to fatigue symptoms, impact of treatment on perceived QoL, and attitudes towards life. In order to deal with the still remaining large amount of extracted data, two of the authors (MW, AT) condensed extracted transcripts of the 'comments' into core texts. For each patient, the data (i.e. think aloud combined with scores and core texts of comments) were organized per interview in one mind map (see example in Fig. 1) by means of the computer package Mindjet Mindmanager Pro 6 (http://www.mindjet.com).

Furthermore, a different mind map was made to organize think aloud data related to the question 'were you tired' for all patients per response category per assessment, including then-test. For the analysis, three authors (MW, AT, TH) each independently read the mind maps of each patient. They studied patients' scores, their think aloud responses, and examined whether response shift type explanations would be provided: recalibration (i.e., using different standards of comparison to assess fatigue over time), reprioritization (i.e., changes in the importance attached to fatigue over time) and reconceptualization (i.e., changes in the meaning of fatigue over time). Two researchers (MW, AT) searched for additional explanations in the core texts to account for the response behaviour and the discrepancies. The research team (MW, AT, TH, MS) discussed critically the different response strategies used by the patients and the robustness of the interpretations of response shift.

\section{Results}

Patients with or without discrepancies

Of the 23 patients, 15 (5 male and 10 female, age 4672) showed discrepancies at least at one measurement 
Fig. 1 Analysis by means of a mind map. Branch EORTC: scores of GH/QOL and the fatigue scale are organized per interview and complemented by the think aloud data. Branch Comments: core texts of relevant parts of transcripts are organized per code and per interview. Note: $(+)$ not all branches of the different interview moments are shown

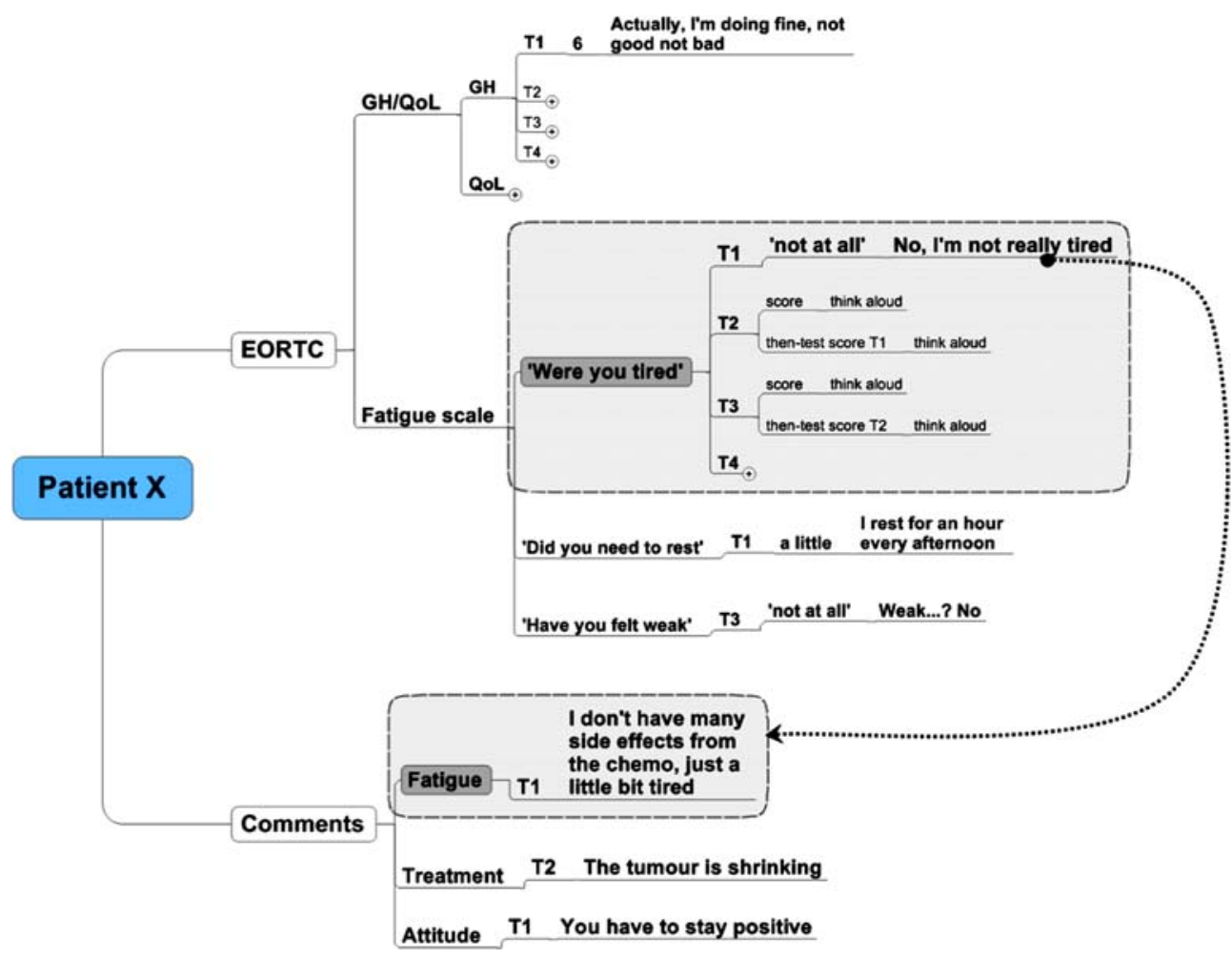

point, i.e. differences between their answer to the EORTC question 'were you tired' and their level of fatigue spontaneously reported during the interview. In their answers to the EORTC questionnaire they all presented themselves positively and said that they were not tired. Mary, for example, was 60 years old at the time of the first interview. During all her interviews she reported that she was tired, but she consistently answered 'not at all' to the EORTC question (Box 1). Only once she scored 'a little bit'. This was at T2 when filling in her questionnaire as a then-test for T1 and she commented " a little, more than at the moment".

Box 1 Example of a patient with discrepancies in reported level of fatigue

\section{Patient Mary \\ Mary was 60 years old and married. She had two sons and two grandchildren. Her answer to the EORTC question was consistently 'not at all', except for the then-test concerning the interview T1. This suggests that she did not suffer from fatigue in the week prior to the interviews and that fatigue due to \\ chemotherapy did not have any impact at all on her energy level. But, during the interview she spontaneously provided information that indicated that chemotherapy had an impact on her life and that she regularly suffered from fatigue.}

\section{T1 EORTC score 'not at all'}

Think aloud: Were you tired... no not at all, no, no difference compared to the past

Interview: I'm getting tired at the least little thing
Box 1 continued

\section{T2 EORTC score 'not at all'}

Think aloud T2: Not tired, last week, not at all Think aloud then-test T1: a little, more than at the moment. Interview: According to the doctors, the X-rays were very good. I'm very optimistic, sometimes I'm tired but that's my own fault. I don't have as much energy as I did before I became ill.

\section{T3 EORTC score 'not at all'}

Think aloud: Last week I wasn't tired, it's the second week after my chemo, not at all tired

Think aloud then-test for T2:not at all

Interview: Yes, it was my last cycle of the chemo, I was afraid that I wasn't going to be able to carry on through the treatment. But I managed, okay; I'm tired but apart from that... nothing at all.

\section{T4 EORTC score 'not at all'}

Think aloud: No I wasn't tired last week, not at all

Think aloud then-test for T3:not at all

Interview: Now and then, I'm tired. It's different to before my chemotherapy. Sometimes I'm so tired, so tired, more than in the past. It comes suddenly...in the middle of the day.

For many of our respondents, every new cycle of chemotherapy had a more severe impact on their energy level. Therefore, the highest level of fatigue was to be expected after the 5th and last cycle of chemotherapy, at T3. However, the 15 patients with discrepancies all answered the question 'were you tired' with 'not at all' or 'a little'. For example, during her 
interviews at T2 and T3, Ann reported the growing impact of every cycle of chemotherapy, but her answer to the EORTC question at both interviews was 'a little' (Box 2).

Box 2 Example of a patient using different response strategies, comparison with more sick patients, response shift and selfpresentation

\section{Patient Ann}

Ann was 47 years old and living with a partner. She didn't have any children.

Her scores suggest that the chemotherapy had a slight impact on her energy level during her treatment and a greater impact 6 weeks after completion of the treatment. But, the interview and the think aloud provided information that indicated that the chemotherapy had a growing impact on her life, and the score 'quite a bit' at T4 was the result of bad news (i.e. a recurrence of the tumour). Furthermore, her data show examples of different response strategies, comparison with more sick patients and selfpresentation.

\section{T1 EORTC score 'not at all'}

Think aloud: Were you tired.. in principle I wasn't tired. I was mentally tired, it costs me a lot of energy to talk with my relatives. I think you mean physically tired. You're tired in the sense that you can hardly put one foot in front of the other. That's being tired. No I'm not tired.

Interview: I want to be realistic, think positively. I try not to worry; there is nothing I can do. I don't know when I will die. I get angry when people are surprised...if I say I'm doing fine...it's my decision how I'm feeling.

\section{T2 EORTC score 'a little bit'}

Think aloud: I feel it a little bit, compared to other people who are very sick. So, if I have pain or when I'm tired I say to myself don't complain, so everything I feel, I only feel a little. I'm doing fine.

Think aloud then-test for T1:a little

Interview: Yesterday, I worked for three hours and I was exhausted. I went to bed in the middle of the day in order to be able to show my friend that I'm doing fine. She has trouble in coping...me... having cancer.

\section{T3 EORTC score 'a little bit'}

Think aloud:A little, yes because I have the feeling that $\underline{I}$ was able to get over it

Think aloud then-test for T2: I think that I've said a little last time, it felt a little, but I shift my limit.

Interview: It's a kind of tiredness, I don't know. I've never been like that. To allow yourself to be tired. I think that the story they all tell, that the last cycle of chemo has the most impact, I think that's very very true.

\section{T4 EORTC score 'quite a bit'}

Think aloud: Yes, I was quite a bit tired, but only mentally tired. I have to adjust to the idea of a new course of treatment and radiation. My health is excellent. I'm able to do everything I like, better than 6 weeks ago. But, with all the medicine I'm taking to suppress the epileptic fits... I'm scared, just like after the start of chemo.

Think aloud then-test for T3:a little but I am not really sure, the pain which I had in my ankels made me tired.

Interview: The radiation will make me tired, but it has not started yet, so I am not tired yet.
Patients with discrepancies were identified in both stages of disease and with both treatment regimens, i.e. 12 patients (LD $n=5$, ED $n=7$ ) treated with chemotherapy and $3 \mathrm{LD}$ patients treated with chemotherapy and radiotherapy. During the course of the treatment (T1-T4, 55 interviews) they answered the question "were you tired" 20 times with "not at all, 29 times with 'a little', 4 times with 'quite a bit' and twice with 'very much' (see individual scores per interview in Table 1)

No discrepancies were identified in 8 of the 23 patients ( $\mathrm{LD} n=4, \mathrm{ED} n=4$, age 39-72). They answered the question 'were you tired' at the end of chemotherapy (T3) with 'quite a bit' or 'very much'. During the course of treatment (T1-T4, 28 interviews) these patients answered the question twice with 'not at all', 9 times with 'a little', 11 times with 'quite a bit' and 6 times with 'very much' (Table 1).

Then-test scores were dissimilar with scores of the previous assessment in 25 out of 52 cases, with higher then-test scores in 16 cases. Transcripts showed that patients had difficulty remembering either the previous measurement point and/or their fatigue at that time.

\section{Response strategies for the four response options}

The think aloud texts for the response categories 'quite a bit' and 'very much' were minimal in the entire study population. Only a few patients reacted briefly during the think aloud, e.g. "quite a bit, too tired to keep my eyes open", "yes quite a bit, very tired" and "next week it will be better...very much". The same pattern was found in all patients for all four response options when filling in the questionnaire as a then-test, e.g. " $a$ little bit, I think", "tired then, no" and "very much". However, patients in the group with discrepancies had much more to say in the conventional QoL measurement when choosing the options 'not at all' and 'a little'. During 'think aloud', they seemed to justify the chosen response category. They used various strategies to moderate the impact of fatigue on their life. We summarized their strategies in four categories: (1) I am not tired all the time (e.g. "only in the afternoon"); (2) I am not really tired, it's something else (e.g. " it's the flu"); (3) I have no problems with it (e.g. "I can still cope with it; I don't want to exaggerate"); (4) I am a little bit tired but it is due to something else (e.g. "I didn't have a proper meal").

Many of our respondents said that they had expected to become very tired as a result of the treatment, but that they were not as sick as they had expected. They were very happy that they were able to 
Table 1 Individual (then-test) scores of patients answering the EORTC QLQ-C30 question 'were you tired'. Response categories 1,2, 3 and 4 are representing respectively the category 'not at all', 'a little', 'quite a bit' and 'very much'. Small-cell lung cancer patients $(n=23)$, limited (LD) and extended (ED) disease receiving 1 st line chemotherapy were interviewed at equivalent points in treatment: at start of chemotherapy (T1), 4 weeks later
(T2), at end of chemotherapy (T3), and 6 weeks later (T4). T1t, $\mathrm{T} 2 \mathrm{t}$ and $\mathrm{T} 3 \mathrm{t}$ are representing then-test scores obtained at respectively $\mathrm{T} 2, \mathrm{~T} 3$ and $\mathrm{T} 4$, when patients are asked to provide a renewed evaluation of their fatigue at the previous assessment. Two groups were identified: patients with $(n=15)$ and without $(n=8)$ discrepancies between their questionnaire answer and fatigue spontaneously reported in the interview

\begin{tabular}{|c|c|c|c|c|c|c|c|c|c|c|}
\hline $\begin{array}{l}\text { Patier } \\
\text { Nr. }\end{array}$ & $\begin{array}{c}\text { aracteristi } \\
\mathrm{M} / \mathrm{F}\end{array}$ & Age & $\mathrm{LD} / \mathrm{ED}$ & $\mathrm{T} 1$ & $\mathrm{~T} 1 \mathrm{t}$ & $\mathrm{T} 2$ & $\mathrm{~T} 2 \mathrm{t}$ & $\mathrm{T} 3$ & $\mathrm{~T} 3 \mathrm{t}$ & $\mathrm{T} 4$ \\
\hline \multicolumn{11}{|c|}{ Discrepancies } \\
\hline P 02 & Male & 57 & ED & 3 & 3 & - & - & 1 & - & - \\
\hline P 04 & Female & 50 & LD & 1 & 2 & 2 & 3 & 2 & 3 & 2 \\
\hline P 08 & Female & 69 & ED & 2 & 2 & 1 & 2 & 1 & 2 & 2 \\
\hline P 09 & Male & 66 & ED & 3 & 2 & 2 & 2 & 2 & 2 & 2 \\
\hline P 10 & Male & 46 & LD & 2 & 2 & 2 & 2 & 2 & 2 & 2 \\
\hline P 12 & Female & 47 & LD & 1 & 2 & 2 & 2 & 2 & 2 & 3 \\
\hline P 15 & Female & 69 & LD & 1 & 2 & 2 & 3 & 2 & 3 & 2 \\
\hline P 17 & Female & 64 & ED & 2 & 2 & 2 & 2 & 2 & - & - \\
\hline P 18 & Male & 72 & ED & 1 & 1 & 2 & - & 1 & - & 3 \\
\hline P 21 & Male & 69 & ED & 2 & 2 & 2 & 3 & 2 & 2 & 2 \\
\hline P 22 & Male & 55 & LD & 4 & 1 & 1 & - & 2 & - & - \\
\hline P 24 & Female & 56 & LD & 1 & 1 & 1 & 2 & 1 & 2 & 1 \\
\hline P 26 & Female & 59 & LD & 4 & 2 & 1 & 2 & 2 & - & - \\
\hline P 32 & Female & 60 & LD & 1 & 2 & 1 & 1 & 1 & 1 & 1 \\
\hline P 34 & Female & 51 & LD & 1 & 1 & 2 & 1 & 2 & 1 & 1 \\
\hline \multicolumn{11}{|c|}{ No discrepancies } \\
\hline P 01 & Female & 42 & LD & 2 & 2 & - & - & 4 & 4 & 3 \\
\hline P 03 & Female & 64 & ED & 3 & 1 & - & - & 3 & 3 & 4 \\
\hline P 13 & Male & 72 & ED & 3 & 3 & 2 & 3 & 3 & & 2 \\
\hline P 14 & Male & 39 & LD & 4 & 3 & 2 & 2 & 3 & - & - \\
\hline P 16 & Male & 68 & LD & 1 & 1 & 3 & 3 & 3 & 3 & 2 \\
\hline P 20 & Female & 44 & LD & 1 & - & 4 & - & 4 & - & 4 \\
\hline P 27 & Male & 69 & LD & 3 & 2 & 2 & - & - & 4 & 2 \\
\hline Р 29 & Male & 63 & ED & 2 & 3 & 3 & 2 & 3 & 3 & 2 \\
\hline
\end{tabular}

cope with the treatment, and had adjusted to the situation. A male patient, for example, had a score of 'not at all' at T2, and explained: "I'm currently doing nothing, so I'm not tired". The respondents indicated that they wanted to be honest when filling in the questionnaire and did not want to lie or to exaggerate their fatigue and, because many patients were not tired all the time they considered a score of 'not at all' or 'a little' to be a suitable score (Box 3).

Box 3 Examples of different response strategies used by patients with discrepancies $(n=15)$

\section{Think aloud about the question 'were you tired'}

I'm not tired all the time

I'm only tired in the afternoon

No not at all, I'm not tired at the moment, it comes suddenly I have to be honest, sometimes I'm tired, I can't say not at all, otherwise I would be lying

I'm not really tired, it's something else

No not tired, it's the flue, that's why I'm tired

It's not being tired you know, it's more like being restless

I'm not physically tired, I'm mentally tired
Box 3 continued

Actually, I can't be tired because the Hb level in my blood is okay

I've no problems with it

I'm currently doing nothing, so I've no problems, I'm not tired Of course, you can make yourself tired, but I' don't

I can still cope with it; I don't want to exaggerate

I'm a little bit tired but it's due to something else A little, but it was my own fault, I did too much I didn't have a proper meal, that's why I was tired I didn't have my lady working for me in the house, she went on holiday

It's because I've got problems with my voice caused by the radiation

\section{Optimism}

Most patients told the interviewer regularly that they had adapted to the situation and had changed their attitude towards a more optimistic perspective. They were not hopeful immediately after diagnosis, but optimism about recovery increased when the tumour was shrinking. Of the 23 patients 17 reported sponta- 
neously that they were optimistic: e.g. "I've got good news, I'm as optimistic as can be", "I'm full of hope because I was diagnosed in an early stage, so I'm good in time". Furthermore, they said that they felt better off than expected, compared to patients who were worse off: e.g. "I'm lucky not to be very sick, compared to the patients I saw at the hospital". Although they experienced the impact of every new cycle of treatment as more severe, they said to be able to cope with the treatment and to accept the side-effects: e.g. "it's part of the package, I'm willing to put up with, knowing the chemo is doing the job properly". Some patients were actually feeling better after each cycle and happy that they were still alive: e.g. "It was much worse than I wanted to admit last time, I'm feeling much better", "I've already a couple of months extra".

In contrast, 5 of the 23 patients expressed pessimistic feelings: e.g. "I'm a broken man, hard work all my life and now...I don't think I've much time left", "I'm a bit depressed, when does it stop, if it doesn't stop it would be better if my life was over", "No plans for the future, you never know when the tumour will come back". These patients all reported high levels of fatigue during the course of the treatment. One patient did not provide specific comments about optimism or pessimism.

\section{Response shift and self presentation}

In both groups-with and without discrepancies-we found patients who had reported to have changed their reference point after $\mathrm{T} 1$ (i.e. recalibration in contrast to T1). They compared their fatigue at the second and following interviews with that of other patients e.g. "I was tired, yes, but compared to the patients I've seen in the hospital, I'm just a little bit tired" or, with the period in which they were more tired (e.g. "Compared to the first week after chemo, it's the second now ... I'm not tired"). In one case, a patient spontaneously re-evaluated her previous measurement: "I told you that I was really tired then, but compared to how I'm feeling now, it was then just a piece of a cake". Another one spoke about a shift of limits which also suggests recalibration: "I already told you that I would change my standards".

We did not find indications of reconceptualization and reprioritization of fatigue. The only exception was Ann who made a distinction between being physically and mentally tired (see Box 2). At T1, she said that she was mentally tired but not physically and her answer was 'not at all': "Tired means that you can hardly put one foot in front of the other". At T4, she said that she had recovered from chemotherapy and was physically able to do anything she wanted but, unfortunately, suffered from sudden epileptic attacks caused by metastases. She was feeling anxious in the same way as at the start of her chemotherapy, and had to consider further treatment options. Just like in the interview at $\mathrm{T} 1$, she said that she was mentally tired but not physically. However, this time her score was 'quite a bit' instead of 'not at all': "Purely, because I was mentally tired last week. I have to adjust to the idea of a new course of treatment. Actually, I have to admit that I really am the cancer patient I never wanted to be". This response pattern might be interpreted as reprioritization (i.e., changes in the importance attached to mental fatigue over time).

Box 4 Examples of coping strategies used by patients with discrepancies $(n=15)$

\section{Spontaneously reported coping behaviour}

Protective behaviour

I'm trying to avoid or minimize pessimistic thoughts I don't think about it, otherwise I can't cope with it We don't talk about it, just follow my every day routine I'm building a wall around myself

Assertive behaviour/power display

I'll show others that I'm managing all right

You have to be positive

You have to believe in yourself, otherwise you can't manage it anymore

Fighting the stigma

I'm not the cancer patient my neighbour thinks I am They think I'm lying on my bed all day

People look at me, and give advice that I don't want

I have to admit that I really am a cancer patient... I didn't want to be (see patient Ann, Box 2).

Because the above mentioned response shift type explanations could not adequately explain our conflicting findings in the discrepancy group we questioned: "Why are patients presenting themselves in the questionnaire more positively than in the informal interview". In our search for an other explanation, we found that 13 of the 15 patients with discrepancies had spontaneously reported how they dealt with having cancer and the perspective of a short life-expectancy. We summarized their comments in three categories (see examples of coping strategies in Box 4): (1) Protective behaviour (e.g. protecting themselves from harmful thoughts); (2) Assertive behaviour/power display (e.g. projecting the image of being positive and managing all right); (3) Fighting the stigma (e.g. fighting against being stigmatized).

Taking these strategies into account, we concluded that a possible mechanism underlying the discrepancies in this group was 'self-presentation'. As the question- 
naires are explicitly related to cancer and since these patients want to distance themselves from being reduced to only a cancer patient, they want to present themselves as a person who just happened to have cancer. Therefore, they applied various strategies to respond to the question on fatigue in order to produce a score that was as favourable as possible and presented themselves as positive and managing their fatigue.

\section{Discussion}

Two third of the patients showed discrepancies in their reported level of fatigue. They reported a gradual decrease in energy at the end of chemotherapy, but they were 'not at all' or just 'a little bit' tired according to their answer to the EORTC questionnaire, with underreporting as a result. They presented a positive image of themselves and used various strategies to explain their choice of response category. A predominant finding was that patients adopted a more optimistic perspective on the treatment. Interestingly, this was not exclusively found in the discrepancy group. The same was true for recalibration and for the only indication of reprioritization. These response shift type explanations did not sufficiently account for the conflicting findings in our discrepancy group.

Self-presentation was found to be an additional (coping) mechanism underlying the discrepancies. Our results suggest that patients are not only concerned about the impression they make on others. They try to protect themselves from negative thoughts and they also feel the need to be positive and to distance themselves from the stereotypical cancer patient. With this strategy they are more capable of coping with a situation that they cannot change.

The suggestion that self-presentation is an underlying mechanism is supported, for example in the case of Ann. After a recurrence of the tumour she adopted the realistic perspective by admitting that she really was 'the cancer patient', which she did not want to be before. It seems that she had given up her attitude of showing others that everything was all right, and for the first time she did not present her self as more positive than she actually was as she did before.

Self-presentation (also called impression management $[6,9]$ ) is a phenomenon described by Leary et al. in relation to health behaviour [10]. They discussed its implications for research in health psychology. Our study shows that, in addition to response shift, selfpresentation may explain unexpected results, at least in SCLC patients. The question 'were you tired' in the EORTC-QLQ-C30 does not unequivocally measure the impact of chemotherapy on the energy level of patients; in fact, with their responses, patients seem to show how that they are managing the situation. From our results we cannot conclude that in the group without discrepancies self-presentation is not present at all, or that whenever self-presentation occurs discrepancies will also be present. However, our study does show that self-presentation affects QoL measurement. These findings must be taken into account when investigating and interpreting QoL data, also in other study populations. Especially after diagnosis and in the initial phase of treatment, self-presentation might be an important coping strategy. In fact, during each phase in which a new equilibrium and a new identity has to be found (e.g. after a recurrence of the tumour, or metastases) self-presentation might affect QoL measurement.

Acknowledgements We wish to thank all the patients for their contribution to this study, which was funded by the Dutch Cancer Society (VU 2000-2339).

\section{Reference}

1. Aaronson, N. K., Ahmedzai, S., Bergman, B., Bullinger, M., Cull, A., \& Duez, N. J., et al. (1993). The European Organization for Research and Treatment of Cancer QLQ-C30: A quality-of-life instrument for use in international clinical trials in oncology. Journal of the National Cancer Institue, 85(5), 365-376.

2. Andrykowski, M. A., Brady, M. J., \& Hunt, J. W. (1993). Positive psychological adjustment in potential bone marrow transplant recipients: Cancer as a psychosocial transition. Psycho-Oncology, 2, 261-276.

3. Bach, J. R., \& Tilton, M. C. (1994). Life satisfaction and wellbeing measures in ventilator assisted individuals with traumatic tetraplegia. Archives of Physical Medicine and Rehabilation, 75(6), 626-632.

4. Bergman, B., Aaronson, N. K., Ahmedzai, S., Kaasa, S., \& Sullivan, M. (1994). The EORTC QLQ-LC13: a modular supplement to the EORTC Core Quality of Life Questionnaire (QLQ-C30) for use in lung cancer clinical trials. EORTC Study Group on Quality of Life. European Journal of Cancer, 30A(5), 635-642.

5. Breetvelt, I. S., \& Van Dam, F. S. (1991). Underreporting by cancer patients: the case of response-shift. Social Science and Medicine, 32(9), 981-987.

6. Goffman, E. (1959). The presentation of self in everyday life. New York: Doubleday.

7. Groen, H. J. M., The, B.A.M., Sanderman, R., De Vries, E. G. E., \& Mulder, N. H. (1995). "Quality of life in lung cancer patients treated by radiation with and without carboplatin" thesis Groen H. J. M. Exploration of new therapy for lung cancer.

8. Hak, T., Veer van der, K., \& Jansen, H. (2004). The ThreeStep Test-Interview (TSTI): An observational instrument for pre-testing self-completion questionnaires, Rotterdam: Erasmus Research Institute of Management. ERIM Report ERS-2004-029-ORG. 
9. Leary, M. R., \& Kowalski, R. (1990). Impression management: A literature review and two component model. Psychological Buletin, 107, 34-47.

10. Leary, M. R., Tchividjian, L. R., \& Kraxberger, B. E. (1994). Self-presentation can be hazardous to your health: impression management and health risk. Health Psychology, 13, 461-470.

11. Llewellyn-Thomas, H. A., Thiel, E. C., \& Clark, R. M. (1989). Patients versus surrogates: Whose opinion counts on the ethics review panels? Clinical Research, 37, 51-55.

12. Mays, N., \& Pope, C. (2000). Qualitative research in health care. Assessing quality in qualitative research. BMJ Jan 1; 320 (7226):50-52.

13. O'Boyle, C. A., Hickey, A., Joyce, C. R. B., Browne, J. P., O'Malley, K., \& Hiltbrunner, B. (1993). The schedule for the evaluation of individual quality of life (SEIQOL): Administration manual. Dublin: Royal College of Surgeons in Ireland, Department of Psychology.
14. O’Boyle, C. A., McGee, H., \& Joyce, C. R. B. (1994). Quality of life: assessing the individual. Advances in Medical Sociology, 5, 159-180.

15. Schwartz, C. E., \& Sprangers, M. A. (1999). Methodological approaches for assessing response shift in longitudinal health-related quality-of-life research. Social Science and Medicine, 48(11), 1531-48.

16. Sprangers, M. (1988). Response shift and the retrospective pretest. Den Haag: SVO.

17. Sprangers, M. A., \& Schwartz, C. E. (1999). Integrating response shift into health-related quality of life research: A theoretical model. Social Science and Medicine, 48(11), $1507-1515$

18. Sprangers, M. A., Van Dam, F. S., Broersen, J., Lodder, L., Wever, L., \& Visser, M. R., et al. (1999). Revealing response shift in longitudinal research on fatigue-the use of the thentest approach. Acta Oncologica, 38(6), 709-718. 